\title{
Visual hallucinations and Capgras delirium ina patient with right occipito-temporal ischemic stroke
}

\author{
Alberto Ibáñez Navarro, José Antonio Oliván Usieto, Iván José Salas García, \\ Newton Arza Candia, Teddy Weimar Cordova Irusta \\ University of Zaragoza, Aragon, Spain
}

\begin{abstract}
The ability to recognize an individual's face and determine whether it is familiar or unfamiliar is an adaptive skill that develops during the first few months of life. In patients suffering from Capgras syndrome, this capacity is impaired, temporarily in relation to an acute organic pathology or chronically triggering a generally positive adaptive attitude. Through a real case of an 81-year-old patient who presents a neurological focal point accompanied by visual hallucinations and a disorder in the perception of a relative as "strange"; this peculiar syndrome is introduced, generally underdiagnosed, which can facilitate the location of dysfunctional brain areas and relate it to the rest of higher cognitive functions.

This delirium is an example of how the brain makes a "logical response"; to a serious lack of information connection. We will also talk about several physio-pathological theories in this respect and their relationship with certain acute pathologies and other chronic ones such as schizophrenia or dementia.
\end{abstract}

Keywords: visual hallucinations, Capgras delirium, ischemic stroke

\section{INTRODUCTION}

The capacity of the human being to recognize the face of an individual and to determine whether it is familiar or unknown is not present from birth, is developing and perfecting throughout the years of life. a nd it is this ability to connect visual information with the emotional memory it triggers that is affected in Capgras patients.

Next, through the introduction of a real case, we propose to develop this peculiar delirium, generally underdiagnosed, which can, on the one hand, facilitate the location of lesions or dysfunctional areas in the context of an organic pathology; and, on the other hand, better understand how the human brain works through the evaluation of higher cognitive functions than those we usually explore.

For its development the ethical norms were fulthe ethical committee of the Sector of Alcañiz did not put inconveniences to its publication and the informed consent of the patient was collected in writing for the publication of the clinical case.

\section{DESCRIPTION OF THE CASE}

This is an 81-year-old woman referred for acute episode of confusion with false recognition of the environment and her daughter for four hours.

Relevant antecedents include hypertension of control with four antihypertensives, diabetes mellitus type II of years of evolution in treatment with oral antidiabetics and mixed hyperlipidemia. Independent for basic activities of daily living. No traits of depression or anxiety or psychiatric disorders.

\section{Exploration}

s he was thermodynamically stable. Glasgow s cale 15, MMse (Mini Mental s tate e xamination) 28 points. NIHss scale (National Institute of Health 
s troke s cale): 3 points. Oriented on the three spheres (person, time, space). To the exploration it presented homonym left hemianopsia accompanied by left sensory extinction. The strength and coordination of both sides were preserved; and the left cutaneous-plantar was indifferent. The rest of the general exploration didn't reveal any more information.

s he also referred to visual hallucinations in the left hemicampus and a false recognition of his daughter as a stranger. "s he looks like my daughter, but I'm sure she's not. s he worries about me, but doctor, she's not my daughter". The rest of the

Brain CT (tomography computerized) showed right occipito-temporal hypodensity with discrete signs of chronic brain atrophy Neither the initial general emergency analysis (biochemistry, ionogram, hemogram, urinary sediment, toxicological study) nor the in-depth study during admission (thyroid hormones, vitamin B12, folic acid, proteinogram, calcemia and phosphatemia) indicated rations.

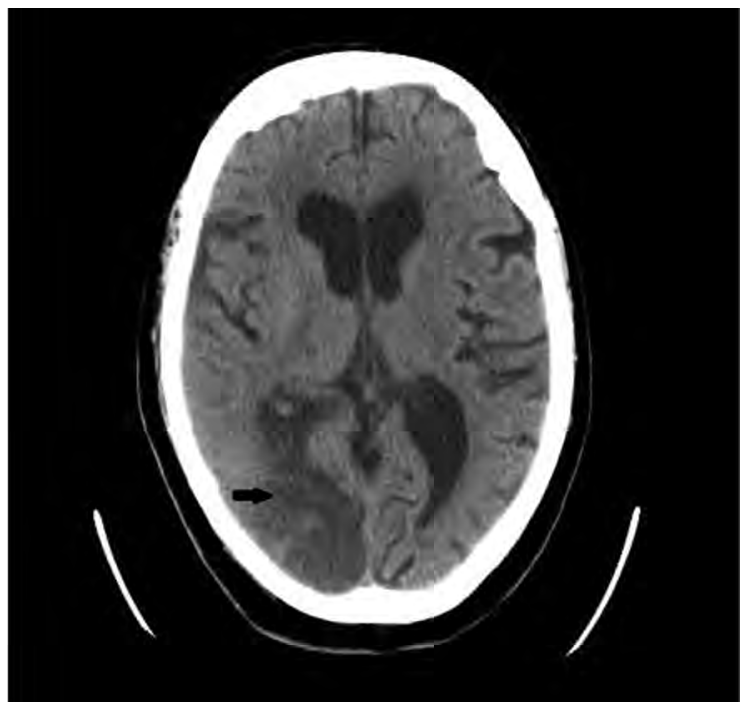

FIGURE 1. Right occipito-temporal hypodensity

Within a few days of admission, neurological symptoms, hallucinations and alterations of perception disappeared and she was discharged asymptomatically. In the subsequent controls by Neurology did not recur either.

The episode was labeled a right occipito-temporal ischemic stroke associated with an acute delirious syndrome.

\section{DISCUSSIONS}

Capgras syndrome is a small, well-organized delirium in which the patient believes that a person, usually a family member or close friend, has been replaced by an impostor identical to that person.

It is usually sudden onset and transitory, although there are also described cases of chronic or episodic character. They are related to a scarce emotional component, which is generally positive which triggers an adaptive behavior to said delirium (1). a lthough they are the least known, the best known are those who ended up in divorces, or homicides. And , this delirium usually co incides with other hallucinations, both visual and auditory, or other failure syndromes - such as Fregoli syndrome, intermetamorphosis or déjà-vu.

It frequently occurs in paranoid schizophrenia, but in $25-40 \%$ it can appear in relation to organic damage such as dementia - 2-30\% in a lzheimer's disease; $8-17 \%$ Lewy body disease; epilepsy or cerebrovascular disease.

Do not forget to rule out organic pathology (2) that can lead to or facilitate it such as severe anemia, electrolyte disorders, vitamin endocrine pathology, malnutrition or dehydration, intoxications, infections or septic processes, cerebrovascular pathology, etc.. a good anamnesis must be carried out, addressing the psycho-neurological spectrum, environment and socio-familiar situation and a previous functional evaluation (examples, Rankin scale, Barthel index, Lawton and Brody scale, functional independence measurement scale, Hamilton scale for depression or Montgomery scale, quality of life scale...).

The question that this pathology evokes is: Is everything we perceive real? since the times of Platon and a ristotle of a ncient Greece, man has been searching for the keys to consciousness and yet all attempts remain mere conjectures and approximations... it is in the twentieth century that it is discovered that the visual pathway is the one that can give us better information about how our psyche works.

We highlight Bruce and Young and Burton's "facial processing model" (3) in which they two fundamental components located in the right temporal lobe, more in the fusiform 
gyrus and the perihypocampic and amygdala areas; these are (4): 1) facial recognition units (FRU), which act as a memory store for previously known faces, 2) and personal identity nodes (PINs) which store emotional memory relating to people/animals/known things/places that has been acquired in multiple contexts and integrated into a single representation that is stored in memory.

The visual pathway is intact ("that's my daughter's face") but the channel of emotional vision, which is a distinct anatomical pathway - parietal right - , does not allow connecting the information of the face with the associated emotional component ("inside me I'm sure it's not my daughter") $(5,6)$. a ny computer would understand it as a system failure and block itself, but the brain, which cannot afford it, decides to give the control to the left cerebral hemisphere, which interprets it as an impersonation ("ergo is someone who has disguised himself as my daughter").

It is a clear example that all illnesses have two dimensions: psychological and physical; and that there is a line between neurology and psychia -

\section{REFERENCES}

1. Hillers Rodriguez R, Madoz Gurpide A, Tirapu Ustarroz J. Propuesta de una batería neuropsicológica para la exploración del síndrome de Capgras. Rev Esp Geriatr Gerontol. 2011.

2. Madoz Gurpide A, Hillers Rodriguez R. Delirio de Capgras: Una revisión de las teorías etiológicas. Rev Neurol 2010;50:420-30.

3. Butler C, Zeman AZJ. Neurological syndromes which can be mistaken for psychiatric conditions. J Neurol Neurosurg Psychiatry 2005;76(Suppl I):31-38.

4. Hirstein W, Ramachandran VS. Capgras Syndrome: A novel probe for understanding the neural representation of identity and familiarity of persons. Proc. R. Lond. B. 1997;264:437-444. try. The more we know about how our brains work, the more we realize how much we have to discover and study.

\section{CONCLUSIONS}

In the event of an atypical symptom or a symptom that the family as different from the usual, an in-depth study must be initiated to rule out the causative organic pathology. It is important to know the previous functional, emotional and nutritional basal state. It is usually a transitory disorder that after solving the triggering cause is resolved without sequelae or risk of recurrence. But it can also be chronicled for years going unnoticed by the adaptive attitude incurred by the mourner.

\section{Acknowledgments}

We would like to thank the emergency physicians, internists, the neurology service and all primary care physicians who care for the health of the community for their work.

Conflict of interest: none declared Financial support: none declared

5. Shah NJ, Marshall JC, Zafiris $O$ et al. The neural correlates of person familiarity. A functional magnetic resonance imaging study with clinical implications. Brain 2001;124:804-815.

6. Hudson AJ, Grace GM. Misidentification syndromes related to face specific area in the fusiform gyrus. J Neurol Neurosurg Psychiatry 2000;69:645-648. 